\title{
Factors Associated with Use of Guideline in Home Management of Malaria among Children in Rural South West Nigeria
}

\author{
Adekunle Akerele, ${ }^{1}$ Oyindamola B. Yusuf, ${ }^{1}$ Catherine O. Falade, ${ }^{2}$ \\ Ikeoluwapo O. Ajayi, ${ }^{1}$ and Franco Pagnoni ${ }^{3}$ \\ ${ }^{1}$ Department of Epidemiology, Medical Statistics and Enviromental Health, College of Medicine, University of Ibadan, Ibadan, Nigeria \\ ${ }^{2}$ Department of Pharmacology and Therapeutics, College of Medicine, University of Ibadan, Ibadan, Nigeria \\ ${ }^{3}$ UNICEF/UNDP/World Bank/WHO Special Programme for Research and Training in Tropical Diseases (TDR), \\ 1211 Geneva, Switzerland
}

Correspondence should be addressed to Oyindamola B. Yusuf, bidemi_yusuf@yahoo.com

Received 13 January 2011; Revised 18 April 2011; Accepted 26 April 2011

Academic Editor: Polrat Wilairatana

Copyright ( $\odot 2011$ Adekunle Akerele et al. This is an open access article distributed under the Creative Commons Attribution License, which permits unrestricted use, distribution, and reproduction in any medium, provided the original work is properly cited.

The dosage regimen for artemether-lumefantrine which is the standard of care for malaria in most of Sub-Saharan countries requires use of treatment guidelines and instructions to enhance caregivers' performance in the treatment of malaria. As part of a larger study evaluating its effectiveness in a rural local government area in southwestern Nigeria, 552 caregivers whose children had fever two weeks preceeding the survey were recruited. Information was collected with interviewer administered questionnaire. A multilevel logistic regression model was fitted using the gllamm approach in Stata to determine the factors associated with use of guideline. Age and educational background of caregiver were significantly associated with guideline use. Caregivers aged 2630 years were 4 times more likely to use guideline than those aged $>40$ years. Caregivers with primary education were 4 times more likely to use guideline compared with caregivers with no formal education. Between-village variance was $0.00092 \pm 0.3084$. Guideline use reduced with increasing age and lower education.

\section{Background}

Malaria remains an important public health problem and a principal cause of childhood mortality. It is estimated that at least 10 percent of all childhood deaths are due to malaria [1]. Mortality is highest among children under five years of age who do not receive prompt/appropriate treatment [1]. Despite the fact that the main strategy for reducing childhood mortality and morbidity is presumptive treatment of all fevers in children with antimalarial drugs [2], the greatest challenge of malaria treatment still occurs during treatment as many children with malaria are treated at home [3].

Emphasis on prompt treatment and distribution of prepackaged antimalarial drugs are strengths of the Home Management of Malaria [4], a strategy proposed by the World Health Organization in an effort to improve prompt access to treatment. This is predicated on the observation that the majority of fevers in that age group in Sub-Saharan Africa are due to malaria [5]. The need to reduce malaria morbidity and mortality through the improvement of home treatment of childhood fevers has led to a number of community-based initiatives, including the training of mothers, community health workers, or shopkeepers in diagnosis, appropriate antimalarial use, and referral [6].

It is very important to help the caregivers in overcoming the problems associated with prompt and effective treatment. A study by NetMark Africa Regional Malaria Program showed that the core of the home management of malaria is the production of effective information, education and communication (IEC) materials, to improve health seeking behaviour, use of prepacks, ensure referral of severe cases to health workers, and so on. It has been shown that the use of treatment guidelines significantly improved the correctness of caregivers' treatment of malaria [7]. Providing guideline (instructions) for caregivers to help manage malaria at home, 
will help the caregivers to perform better in the treatment of the disease.

Ajayi et al. also reported a significant increase in correct use of chloroquine from $2.6 \%$ at baseline to $52.3 \%$ after an intervention which provided treatment guidelines and taught mothers treating their children at home in its use of the treatment compared with mothers in the control group [7]. China achieved a 95\% reduction in malaria incidence between 1970 and 1990 following the launch of its National Malaria Control Program [8]. Provision and enforcement of treatment guideline is part of the control program, and it is believed to have contributed in a large measure to achieving this remarkable outcome. Against the backdrop of evidence indicating the beneficial effect of the use of treatment guideline for the treatment of malaria, this study aims to determine the factors that are associated with use of guidelines in home management of childhood malaria among caregivers using a multilevel logistic modeling approach that accounted for the hierarchical nature of the data.

\section{Methods}

2.1. Study Area. This study was carried out in Ona Ara Local Government Area, one of the 36 LGAs in Oyo state in south western Nigeria as part of a larger study on improving home management of malaria using artemether-lumefantrine (AL) in rural south-west Nigeria among children aged 4 months5years [9]. Ona Ara LGA has 8 health districts comprising 6 rural and 2 urban. Two rural communities were selected using simple random sampling comprising 56 communities. In Ona Ara LGA, a primary health center is usually attached to about 12 communities. Thirty communities were selected from this LGA using probability proportional to size. This study utilized the data extracted from a larger study evaluating the effectiveness of artemether-lumefantrine in a rural local government area in southwestern Nigeria $[9,10]$.

The study utilized community-based drug distributors (CBDs) consisting of mother trainers (mothers trained to identify and manage incidence of fever), health workers and patent medicine sellers (drug sellers in the communities) who were trained on the use and administration of AL. As part of the home management of malaria strategy, the caregivers visited the community-based distributors when their children had fever. During the interaction, caregivers collected AL and a pictorial treatment guideline after due explanation of its use. The study population consisted of caregivers in all the communities studied who had children aged 4-59 months with fever in the two weeks preceding the survey and consented to participate in the study. Research assistants who had received a three-day training interviewed one caregiver per household. In cases where more than one caregiver met the inclusion criteria, the caregiver to be interviewed was selected by balloting. The larger study received ethical approval both from the WHO ethical review committee and the Oyo state ministry of health ethical committee. Details of the methodology including the sample size calculation have been previously described by Ajayi et al. $[9,10]$.
2.2. Data Set. The data used for this analysis was a total of 552 records of fever cases from 30 villages extracted from the larger study on HMM using artemether-lumefantrine demonstrating the hierarchical structure of the data. The data was analyzed using SPSS 16 and Stata 10.0 statistical packages $[11,12]$. Data was checked for errors and cleaned. Variables used in this analysis include sociodemographic factors, designation of persons who took treatment decision, and use of guideline.

2.3. Data Analysis. Frequency tables and graphs were used to present relevant variables. Descriptive statistics such as means, medians, and standard deviations were used to summarize quantitative variables while qualitative variables were summarized with proportions. The Chi-squared test was used to investigate association between categorical variables. Variables significant in the bivariate analysis were then entered into a multilevel logistic regression model to predict the strength of the association between the dependent variable "did you use guideline for treatment when child was sick?" and the independent variables. All of the analysis was done at the $5 \%$ level of significance.

2.4. Multilevel Modelling. The outcome variable "did you use guideline for treatment when child was sick?" is a binary variable (use guideline: yes/no). Let $\pi_{i}$ be the predicted probability and $\pi_{i} /\left(1-\pi_{i}\right)$ the odds of using guideline for treatment in an episode of malaria for the $i$ th individual. The conventional logistic regression is given by

$$
\operatorname{logit}\left(\pi_{i}\right)=B_{0}+B_{1} X_{1}+\cdots+B_{p} X_{p},
$$

where $B_{0}$ is the intercept and $B_{1}, \ldots, B_{p}$ are the regression coefficients of the independent variables $X_{1}, \ldots, X_{p}$. The odds ratio (OR) associated with the variable $X_{1}$ is the exponential function of its parameter $B_{1}\left(\mathrm{OR}_{X_{1}}=\exp \left(B_{1}\right)\right)$.

In this analysis, a multilevel logistic model was fitted on the outcome variable "did you use guideline for treatment when child was sick?" and the sociodemographic variables significant in the bivariate analysis. Two levels of organization (individual and village) were stated in the multilevel model. Let $\pi_{i v}$ be the predicted probability of using guideline treatment in an episode of malaria, by the $i$ th individual in the $v$ th village. The general equation of a multilevel logistic model is

$$
\operatorname{logit}\left(\pi_{i v}\right)=B_{0 v}+B_{1} X_{1 i v}+\cdots+B_{p} X_{p i v}+u_{0 v} .
$$

The difference between conventional (single level) and multilevel models is the structure of the random part of the model which is also called residual variation error. In the conventional model, there is only one level and the structure of the residual error is reduced to one value the individual level residual variance, while, in the multilevel model, the structure of the random part (residual variation) is more complex and partitioned among levels of the hierarchy. The random part of the logistic model is partitioned among an individual level variance (which is set to be binomial) and village level variance. 
TABle 1: Demographic characteristics of caregiver.

\begin{tabular}{|c|c|c|}
\hline Characteristic & Frequency & Percentage \\
\hline \multicolumn{3}{|l|}{ Age of caregiver (years) } \\
\hline $18-25$ & 138 & 25.1 \\
\hline $26-30$ & 169 & 30.7 \\
\hline $31-35$ & 109 & 19.8 \\
\hline $36-40$ & 84 & 15.7 \\
\hline$>40$ & 46 & 8.4 \\
\hline No response & 3 & 0.5 \\
\hline Total & 552 & 100 \\
\hline \multicolumn{3}{|l|}{ Sex of caregiver } \\
\hline Male & 23 & 4.2 \\
\hline Female & 529 & 95.8 \\
\hline Total & 552 & 100 \\
\hline \multicolumn{3}{|l|}{ Marital status of caregiver } \\
\hline Single & 6 & 1.1 \\
\hline Married & 473 & 85.7 \\
\hline Separated & 45 & 8.2 \\
\hline Widowed & 19 & 3.4 \\
\hline Divorced & 5 & 0.9 \\
\hline No response & 4 & 0.7 \\
\hline Total & 552 & 100 \\
\hline \multicolumn{3}{|c|}{ Educational status of caregiver } \\
\hline Primary & 276 & 50.4 \\
\hline JSS/Mid & 67 & 12.2 \\
\hline $\mathrm{SSS} / \mathrm{Sec}$ & 33 & 6.0 \\
\hline Tech/Comm/Voc & 3 & 0.5 \\
\hline University & 4 & 0.6 \\
\hline No formal education & 17 & 3.1 \\
\hline None & 147 & 26.7 \\
\hline No response & 5 & 0.9 \\
\hline Total & 552 & $100 \%$ \\
\hline \multicolumn{3}{|c|}{ Main occupation of caregivers } \\
\hline Unemployed & 2 & 0.4 \\
\hline Farming & 220 & 39.9 \\
\hline Artisan & 49 & 8.9 \\
\hline Trading & 252 & 45.7 \\
\hline Apprenticeship & 3 & 0.5 \\
\hline Housewife & 17 & 3.1 \\
\hline Others & 3 & 0.5 \\
\hline No response & 6 & 1.1 \\
\hline Total & 552 & 100 \\
\hline
\end{tabular}

From a computational point of view, multilevel modelling can be seen as a two-stage process. First, a separate individual level regression is defined for each village. Then, each of the village-specific coefficients is modeled as a function of village variables. So multilevel analysis allows the partition of the village-specific coefficients: a fixed part that is common across villages and a random part varying between villages. Intraclass correlation was obtained to measure correlation of
TABle 2: Distribution of use of guideline.

\begin{tabular}{lcc}
\hline Variable & Frequency & Percentage \\
\hline Ever used guideline & 365 & 66.1 \\
Never used guideline & 27 & 4.9 \\
Did not have guideline & 160 & 29.0 \\
\hline Total & 552 & 100.0 \\
\hline Caregivers & Never used guideline & Used guideline \\
\hline Mother & $4(1 \%)$ & $107(29.3 \%)$ \\
Father & 0 & $29(7.9 \%)$ \\
Grandmother & 0 & $6(1.6 \%)$ \\
Health worker & $6(1.6 \%)$ & $27(7.4 \%)$ \\
Mother trainer & $12(3.1 \%)$ & $135(37.0 \%)$ \\
Patent medicine seller & $5(1.3 \%)$ & $54(14.7 \%)$ \\
No response & 0 & $7(1.9 \%)$ \\
\hline Total & $27(7 \%)$ & $365(93 \%)$ \\
\hline
\end{tabular}

use of guideline by individuals from different villages. Coefficients in the models were estimated using a Second-Order Penalised Quasi likelihood (PQL). Statistical significance of parameters was tested using Wald Statistic. The multilevel model was fitted using the gllamm module in Stata. In this analysis, both the multilevel logistic and the conventional logistic models were conducted and outputs were compared.

\section{Results}

Mean age of caregivers was $32 \pm 9.6$ years, and over $95 \%$ were females while $473(85.7 \%)$ were married. The majority were traders, $252(45.7 \%)$. Further details of the demographic characteristics of caregivers are shown in Table 1.

3.1. Use of Guideline. Of the 552 respondents, 392 (71.0\%) caregivers reported that they still had the guideline. Of these, 365 caregivers (93\%) used the guideline correctly while $27(6.9 \%)$ did not use guideline. Of those who used the guideline, 135 (35.1\%) were mother trainers, 107 $(27.8 \%)$ were mothers and only $6(1.6 \%)$ were grandmothers (Table 2).

3.1.1. Bivariate Analysis of Factors Associated with Use of Guideline for Treatment. Guideline use appeared to increase with increase in age except in the 18-25 age categories. A total of $111(28.4 \%)$ respondents aged $26-30$ yrs used the guideline while $86(22.0 \%)$ aged $31-35$ also used it $\left(\chi^{2}=\right.$ $15.268, P<0.05)$. More females used the guideline compared to males, $363(85.6 \%)$ versus $9(4.0 \%)\left(\chi^{2}=0.151, P>0.05\right)$. About two thirds of caregivers who used the guideline 199 $(66 \%)$ had primary education, $80(26.6 \%)$ had postprimary education, and $8(2.7 \%)$ had no formal education $\left(\chi^{2}=\right.$ $6.225, P<0.05)$ (Table 3).

3.1.2. Multilevel Logistic Regression Modelling of Factors Associated with Guideline Use. In order to account for the hierarchical nature of the data as it affects guideline use, 
TABLE 3: Guideline use by demographic characteristics of caregivers.

\begin{tabular}{|c|c|c|c|c|c|}
\hline & Yes & No & Total & $\chi^{2}$ & $P$ value \\
\hline \multicolumn{6}{|l|}{ Age } \\
\hline $18-25$ & $95(24.3 \%)$ & $5(1.3 \%)$ & $100(25.6 \%)$ & & \\
\hline $26-30$ & $111(28.4 \%)$ & $4(1.0 \%)$ & $115(29.4 \%)$ & & \\
\hline $31-35$ & $86(22.0 \%)$ & $4(1.0 \%)$ & $90(23.0 \%)$ & 16.592 & 0.002 \\
\hline $36-40$ & $49(12.5 \%)$ & $8(2.0 \%)$ & $57(14.5 \%)$ & & \\
\hline$>41$ & $23(5.9 \%)$ & $6(1.6 \%)$ & $29(7.4 \%)$ & & \\
\hline Total & $364(93.1 \%)$ & $27(6.9 \%)$ & $391(100 \%)$ & & \\
\hline \multicolumn{6}{|l|}{ Sex } \\
\hline Male & $9(4.0 \%)$ & $1(0.7 \%)$ & $10(4.7 \%)$ & 0.151 & 0.698 \\
\hline Female & $363(85.6 \%)$ & $26(6.2 \%)$ & $380(91.8 \%)$ & & \\
\hline Total & $372(93 \%)$ & $27(7.0 \%)$ & $390(100 \%)$ & & \\
\hline \multicolumn{6}{|l|}{ Educational level } \\
\hline Primary education & $199(66.0 \%)$ & $10(3.3 \%)$ & $209(69.3 \%)$ & & \\
\hline Post primary education & $80(26.6 \%)$ & $2(0.7 \%)$ & $82(27.3 \%)$ & 6.25 & 0.05 \\
\hline No formal education & $8(2.7 \%)$ & $2(0.7 \%)$ & $10(3.4 \%)$ & & \\
\hline Total & $287(95.3 \%)$ & $14(4.7 \%)$ & $301(100 \%)$ & & \\
\hline
\end{tabular}

TABLE 4: Basic multilevel model showing the gradual introduction of independent variables.

\begin{tabular}{|c|c|c|c|c|}
\hline Parameter & Categories & & Model coefficient & \\
\hline Fixed part & & Model A & Model B & Model C \\
\hline Intercept & & $3.02(0.27)$ & $2.40(1.04)$ & $1.59(1.13)$ \\
\hline \multirow[t]{5}{*}{ Age } & $18-25$ & & $0.68(1.16)$ & $0.10(1.31)$ \\
\hline & $26-30$ & & $2.13(1.45)$ & $1.43(1.59)$ \\
\hline & $31-35$ & & $0.68(1.20)$ & $-0.05(1.38)$ \\
\hline & $36-40$ & & $-0.61(1.15)$ & $-1.21(1.30)$ \\
\hline & $>41^{*}$ & & & \\
\hline \multirow[t]{3}{*}{ Education } & No formal education* & & & \\
\hline & Primary & & & $1.3691(1.0008)$ \\
\hline & Post primary & & & $2.0643(1.1274)$ \\
\hline \multicolumn{5}{|l|}{ Random part } \\
\hline Village level & & $2.089 \times 10^{-21}$ & $8.027 \times 10^{-19}$ & $3.687 \times 10^{-19}$ \\
\hline Variance & & $\left(3.499 \times 10^{-11}\right)$ & $\left(8.613 \times 10^{-10}\right)$ & $\left(5.57 \times 10^{-10}\right)$ \\
\hline Intravillage correlation & & $6.356 \times 10^{-22}$ & $2.442 \times 10^{-19}$ & $1.122 \times 10^{-19}$ \\
\hline
\end{tabular}

${ }^{*}$ Reference category.

multilevel logistic regression models were fitted. Sociodemographic variables significant in the bivariate analysis which are age and the education level of the caregiver were entered into the model. Table 4 contains basic multilevel models, while Table 5 contains both the multilevel model and the conventional logistic model.

3.2. The Basic Multilevel Models. The equation of the null model in which no variable was introduced is Model A in Table 4 , and it is given by

$$
\operatorname{logit}(i v)=\beta_{0 v}=\beta_{0}+u_{0 v},
$$

where $\beta_{0}=2.60$ is the "average use of guideline," identical for the 30 villages. Thus, the model allows for residual variations about the intercept which quantify differences between use of guideline by each caregiver and in each village. These differences, called village-level residuals and noted $u_{0 v}$, are assumed to be normally distributed, with mean zero. The variance, $\sigma_{0 v}^{2}$, estimation is 0.0009119 in Model A.

In a second stage, age was introduced (Model B), and the equation becomes

$$
\operatorname{logit}(i v)=\beta_{0}+B_{1 i} \text { age }+u_{0 v}
$$

where $\beta_{0}=1.34, B_{1}=1.60, B_{2}=1.97, B_{3}=1.72$, $B_{4}=0.45$, and variance $\left(u_{0 v}\right)=0.0009119$. The odds ratio of the categories of age showed that caregivers in the age group 18-25 were 5 times more likely to use the guideline for treatment compared to caregivers in the age group $>40$ years $(\mathrm{OR}=4.9)$. Also, caregivers aged between 26 and 30 years were about 8 times more likely to use guideline for the 
TABLE 5: Multilevel logistic model and conventional logistic model.

\begin{tabular}{|c|c|c|c|c|c|c|}
\hline \multirow[b]{3}{*}{ Variable } & \multicolumn{3}{|c|}{ Multilevel logistic regression analysis } & \multicolumn{3}{|c|}{ Conventional logistic regression analysis } \\
\hline & \multicolumn{3}{|c|}{ Model D } & \multicolumn{3}{|c|}{ Model E } \\
\hline & Coefficients (Se) & Odds ratio & $95 \% \mathrm{CI}$ & Coefficients (Se) & Odds ratio & $95 \% \mathrm{CI}$ \\
\hline \multicolumn{7}{|l|}{ Age } \\
\hline $18-25$ & $0.1047(1.3056)$ & 1.1104 & $0.0859-14.3488$ & $0.0944(1.3064)$ & 1.098 & $0.084-14.224$ \\
\hline $26-30$ & $1.4277(1.5859)$ & 4.1689 & $0.1862-93.3280$ & $1.4177(1.5869)$ & 4.128 & $0.184-92.570$ \\
\hline $31-35$ & $-0.0457(1.3812)$ & 0.9553 & $0.0638-14.3157$ & $-0.0568(1.3825)$ & 0.945 & $0.063-14.195$ \\
\hline $36-40$ & $-1.2052(1.2906)$ & 0.2996 & $0.0239-3.7598$ & $-1.1789(1.2918)$ & 0.3076 & $0.024-3.870$ \\
\hline \multicolumn{7}{|l|}{$>40^{*}$} \\
\hline \multicolumn{7}{|l|}{ Educational level } \\
\hline Primary & & & & & & $0.565-28.460$ \\
\hline Postprimary & $1.3691(1.0008)$ & 3.9319 & $0.5530-27.9586$ & $1.3890(0.9998)$ & 4.0108 & $0.771-80.749$ \\
\hline No formal education* & $2.0643(1.1274)$ & 7.8797 & $0.7693-80.7119$ & $2.0660(1.1864)$ & 7.8929 & \\
\hline Random effects parameter & Estimate of variance & Standard error & & & & \\
\hline Community of caregiver & $3.687 \times 10^{-19}$ & $5.566 \times 10^{-10}$ & & & & \\
\hline
\end{tabular}

${ }^{*}$ Reference category.

treatment than those of age group $>40$ years $(\mathrm{OR}=7.2)$. On the other hand, caregivers in the age group 31-35 years were 5 times likely to use guideline for treatment compared to those of age group $>40$ years $(\mathrm{OR}=5.6)$. The odds of guideline use is observed to reduce with increasing age of caregivers while the residual variance $\sigma_{0 v}^{2}$ does not differ from that of Model A.

In Model C, educational qualification of caregiver was included, and the model equation becomes

$$
\operatorname{logit}(i v)=\beta_{0}+B_{1} \text { age }+B_{2} \mathrm{edu}_{i}+B_{6} \mathrm{edu}_{i i}+u_{0 v} .
$$

From the respective categorical parameter for age and education of caregiver, the odds ratio of the categories of age showed that caregivers in the age group 18-25 were more likely to use the guideline for treatment compared to caregivers in the age group $>40$ years $(\mathrm{OR}=1.1)$. Also, caregivers aged between 26 and 30 years were about 4 times more likely to use guideline for the treatment than those of age group $>40$ years $(\mathrm{OR}=4.1)$. On the other hand, caregivers in the age group 31-35 years were less likely to use guideline for treatment compared to those of age group $>40$ years $(\mathrm{OR}=0.95)$. Also caregivers with primary education were 4 times more likely to use guideline for treatment of child during episode of malaria compared to caregivers with no formal education $(\mathrm{OR}=3.9)$, while caregivers with postprimary education were 8 times more likely to use guideline for treatment compared to caregivers with no formal education $(\mathrm{OR}=7.9)$. Increasing age appears to be associated with reducing odds ratio of guideline use while increasing education appears to be associated with increasing odds ratio of guideline use but the residual variance $\sigma_{0 v}^{2}$ remained unchanged.

3.3. Multilevel Model and Conventional Logistic Model. The multilevel model showed that caregivers in the age group 18-25 were more likely to use the guideline for treatment compared to caregivers in the age group $>40$ years $(\mathrm{OR}=1.1$,
95\% CI: $0.086-14.35, P=0.936$ ) (Model D). This is similar to the results in the conventional logistic model but with higher standard errors (Model E). Also caregivers with primary education were 4 times more likely to use guideline for treatment of child during episode of malaria compared to caregivers with no formal education $(\mathrm{OR}=3.9$, 95\% CI: $0.5530-27.9586, P=0.171$ ), while caregivers with postprimary education were 8 times more likely to use guideline for treatment compared to caregivers with no formal education $(\mathrm{OR}=7.9,95 \% \mathrm{CI}$ : $0.769-80.712$, $P=0.082)$. These estimates are also slightly different from the ones in the conventional logistic models (Table 5). The estimate of the variance due to the village effect was $3.687 \times 10^{-19} \pm 5.566 \times 10^{-10}$, while the intraclass correlation was $1.122 \times 10^{-19}$.

\section{Discussion}

The results from this study showed that the use of guideline by caregivers in the home management of malaria is influenced by sociodemographic characteristics of the caregiver. In the design of this study health education was provided for caregivers that resulted in a high proportion of use of guideline. This confirms the findings in another study where use of guideline was associated with increased chloroquine use after the caregivers had been educated [7]. In this study, age and educational background of caregivers were the two sociodemograhic characteristics of the caregivers that were associated with use of guideline.

The proportion of caregivers in the younger age group that used the guideline was more than that of those in the older age group. The chance of use of guideline is higher for the younger age group than for the older age group. This implies that the age group of the caregiver should be taken into cognizance when designing guideline for use in malaria treatment. The findings of this study showed that a lower proportion of caregivers in the older age group were willing to use guideline for treatment. This may not be unconnected 
with their previous experience in managing malaria. This finding is similar to the findings of another study where it was reported that older caregivers prefer to make prescriptions themselves and call for formal facility support when the case becomes complicated [13]. This must be taken note of during future interventions so that field workers will pay special attention to this category of caregivers. This would help field workers attain a higher level of success in encouraging the use of guideline for treatment of malaria episode.

Association of educational level of the caregiver with use of guideline is in keeping with findings of previous studies where it has been observed that the education of caregivers was significantly associated with use of guideline $[7,14]$. Caregivers with primary education exhibited four times chance of using the guideline which underscores the importance of educating future and current mothers as this would be of help in improving the home management of malaria strategy and possibly the general health of children and wards. Although the proportion of respondents with no formal education in this study was low, this is necessary so in other population groups or communities in Nigeria or in Sub-Saharan Africa. Free primary school education was introduced as government policy in south-western Nigeria where this study was conducted as far back as 1955 .

In a similar study [15], sociocultural factors were reported as one of the factors that might have influenced malaria treatment. However, this was not so in this study. The results may as well be due to the training the caregivers obtained in the study. Even though the intraclass correlations were low, valid estimates have been drawn through the use of the multilevel models.

\section{Acknowledgments}

This study was part of a larger study that received support from the Implementation Research Unit of the WHO/TDR, Geneva, Switzerland under the grant "Improving Home Management of Malaria Using Artemether-Lumefantrine in Rural Southwestern Nigeria Project ID A41054." The authors are grateful to the community members of Ona Ara Local government for consenting to participate in this study.

\section{References}

[1] World Health Organization, Report of the Expert Committee on Malaria, World Health Organization, 2005.

[2] Federal Ministry of Health (FMOH), "National Malaria Control Programme in Nigeria," Annual Report, 2005.

[3] J. G. Breman, "The ears of the hippopotamus: manifestations, determinants, and estimates of the malaria burden," American Journal of Tropical Medicine and Hygiene, vol. 64, no. 1-2, pp. 1-11, 2001.

[4] H. Hopkins, A. Talisuna, C. J. M. Whitty, and S. G. Staedke, "Impact of home-based management of malaria on health outcomes in Africa: a systematic review of the evidence," Malaria Journal, vol. 6, article 134, 2007.

[5] M. Malimbo, E. Mugisha, F. Kato, C. Karamagi, and A. O. Talisuna, "Caregivers' perceived treatment failure in homebased management of fever among Ugandan children aged less than five years," Malaria Journal, vol. 5, article 124, 2006.
[6] A. F. Oreagba, A. T. Onajole, S. O. Olayemi, and A. F. B. Mabadeje, "Knowledge of malaria amongst caregivers of young children in rural and urban communities in Southwest Nigeria," Tropical Journal of Pharmaceutical Research, vol. 3, no. 1, pp. 299-304, 2004.

[7] I. O. Ajayi, C. O. Falade, E. A. Bamgboye, A. M. J. Oduola, and O. O. Kale, "Assessment of a treatment guideline to improve home management of malaria in children in rural south-west Nigeria," Malaria Journal, vol. 7, article 24, 2008.

[8] Lining and Fee, "Chinese National Malaria Control Program," Tech. Rep., 2000.

[9] I. O. Ajayi, E. N. Browne, B. Garshong et al., "Feasibility and acceptability of artemisinin-based combination therapy for the home management of malaria in four African sites," Malaria Journal, vol. 7, article 6, 2008.

[10] I. O. Ajayi, E. N. Browne, F. Bateganya et al., "Effectiveness of artemisinin-based combination therapy used in the context of home management of malaria: a report from three study sites in sub-Saharan Africa," Malaria Journal, vol. 7, article 190, 2008.

[11] Statistical Package for the Social Sciences SPSS, Chicago, IL, USA.

[12] StataCorp, "Stata Statistical Software: release 10," College Station, TX: StataCorp LP, 2007.

[13] O. O. Chirdan, A. I. Zoakah, and C. L. Ejembi, "Impact of health education on home treatment and prevention of malaria in Jengre, North Central Nigeria," Annals of African Medicine, vol. 7, no. 3, pp. 112-119, 2008.

[14] U. M. Chukwuocha, O. B. Nkwankwo, N. A. Amadi Agwu et al., "Treatment seeking behavior of mothers of febrile children in some rural part of imo state Nigeria, implication for home management of malaria in endemic areas," International Journal of Tropical Medicine, vol. 4, no. 3, pp. 132-135, 2009.

[15] H. A. Mwenesi, Sociocultural and Behavioural Issues in the Treatment and Prevention of Malaria, WHO/TDR, 2008. 


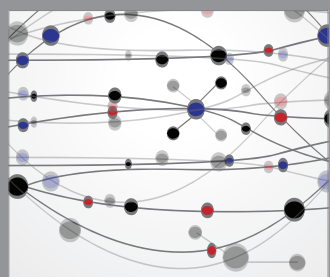

The Scientific World Journal
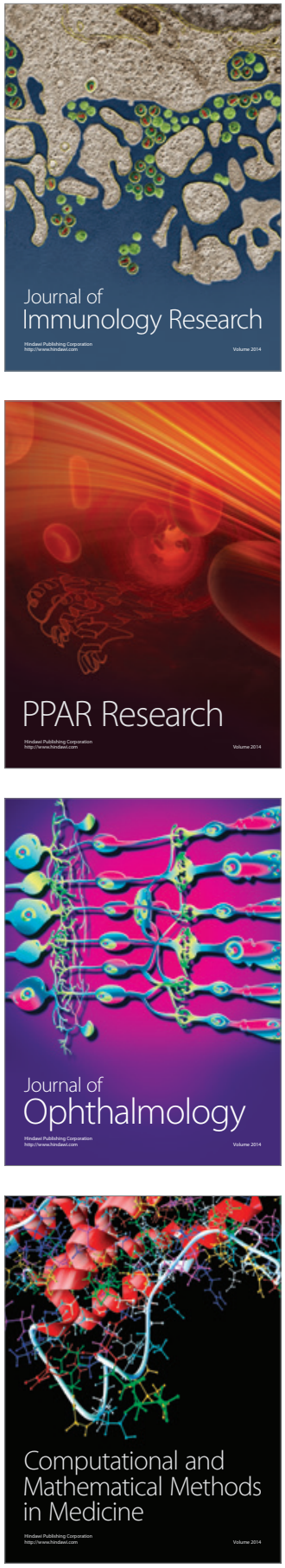

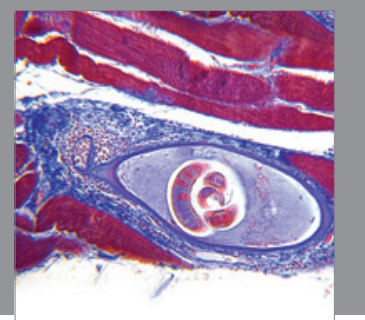

Gastroenterology

Research and Practice
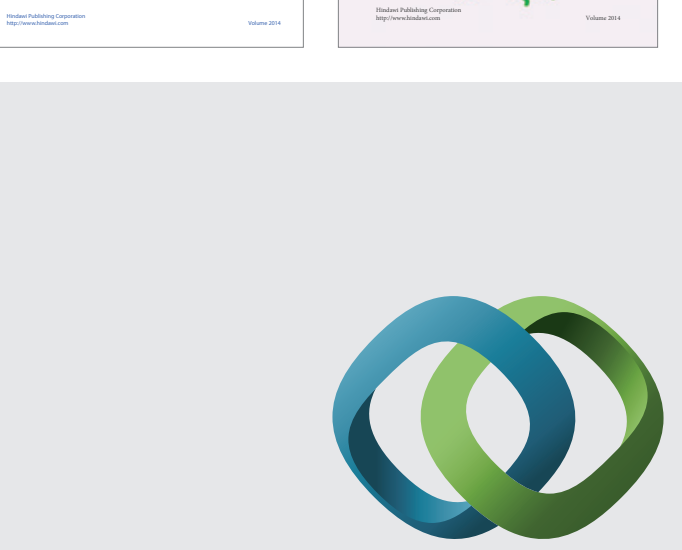

\section{Hindawi}

Submit your manuscripts at

http://www.hindawi.com
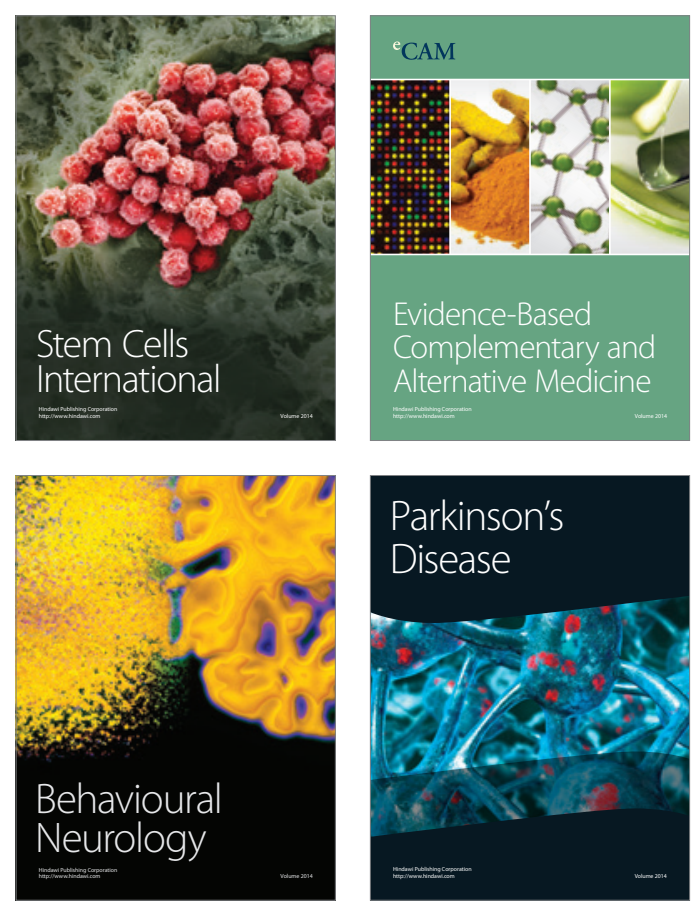

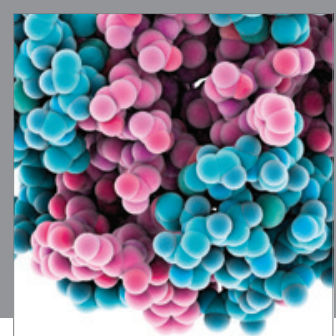

Journal of
Diabetes Research

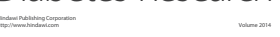

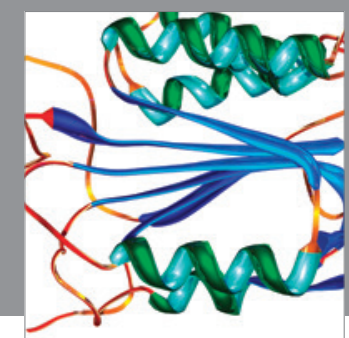

Disease Markers
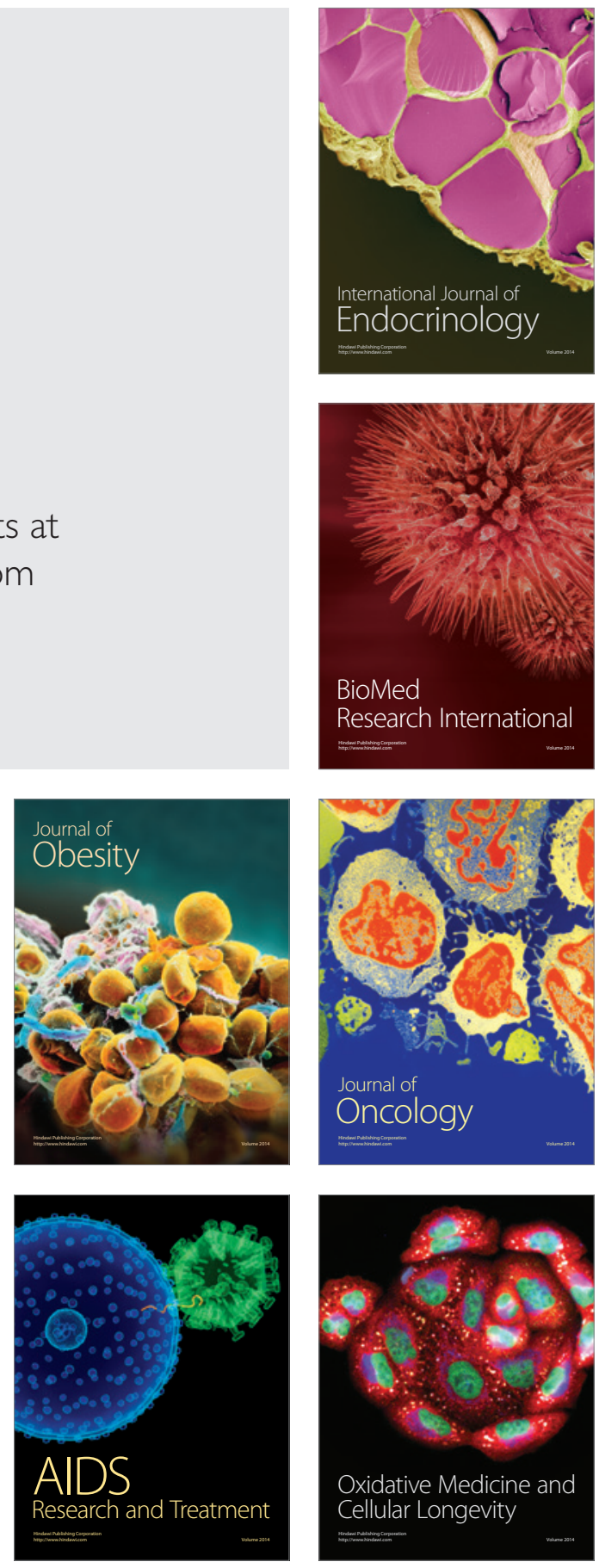